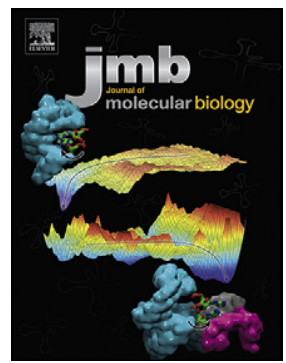

\title{
Molecular Analysis of Two Novel Missense Mutations in the GDF5 Proregion That Reduce Protein Activity and Are Associated with Brachydactyly Type C
}

\author{
Katja Stange ${ }^{1,2}$, Tino Thieme ${ }^{3}$, Karen Hertel $^{3}$, Silke Kuhfahl ${ }^{3}$, \\ Andreas R. Janecke ${ }^{4,5}$, Hildegunde Piza-Katzer ${ }^{6}$, Maila Penttinen ${ }^{7}$, Marja Hietala ${ }^{7}$, \\ Katarina Dathe ${ }^{8}$, Stefan Mundlos ${ }^{1,8,9}$, Elisabeth Schwarz ${ }^{3}$ and Petra Seemann ${ }^{1,2}$ \\ 1 - Berlin Brandenburg Center for Regenerative Therapies, Charité-Universitätsmedizin Berlin, 13353 Berlin, Germany \\ 2 - Berlin Brandenburg School for Regenerative Therapies, Charité-Universitätsmedizin Berlin, 13353 Berlin, Germany \\ 3 - Martin-Luther-University Halle-Wittenberg, Technical Biochemistry, 06120 Halle, Germany \\ 4 - Department of Pediatrics I, Innsbruck Medical University, 6020 Innsbruck, Austria \\ 5 - Division of Human Genetics, Innsbruck Medical University, 6020 Innsbruck, Austria \\ 6 - Department of Plastic, Reconstructive and Aesthetic Surgery, Innsbruck Medical University, 6020 Innsbruck, Austria \\ 7 - Department of Clinical Genetics, Turku University Hospital, 20014 Turku, Finland \\ 8 - Institute for Medical Genetics and Human Genetics, Charité-Universitätsmedizin Berlin, 13353 Berlin, Germany \\ 9 - Research Group Development and Disease, Max-Planck-Institut für Molekulare Genetik, 14195 Berlin, Germany
}

Correspondence to Petra Seemann: Berlin Brandenburg Centerfor Regenerative Therapies, Charité-Universitätsmedizin Berlin, Augustenburger Platz 1, 13353 Berlin, Germany. petra.seemann@charite.de

http://dx.doi.org/10.1016/j.jmb.2014.07.029

Edited by M. Yaniv

\begin{abstract}
Growth and differentiation factor 5 (GDF5) plays a central role in bone and cartilage development by regulating the proliferation and differentiation of chondrogenic tissue. GDF5 is synthesized as a preproprotein. The biological function of the proregion comprising 354 residues is undefined. We identified two families with a heterozygosity for the novel missense mutations p.T201P or p.L263P located in the proregion of GDF5. The patients presented with dominant brachydactyly type $\mathrm{C}$ characterized by the shortening of skeletal elements in the distal extremities. Both mutations gave rise to decreased biological activity in in vitro analyses. The variants reduced the GDF5-induced activation of SMAD signaling by the GDF5 receptors BMPR1A and BMPR1B. Ectopic expression in micromass cultures yielded relatively low protein levels of the variants and showed diminished chondrogenic activity as compared to wild-type GDF5. Interestingly, stimulation of micromass cells with recombinant human proGDF5 ${ }^{\text {T201P }}$ and proGDF5 ${ }^{\text {L263P }}$ revealed their reduced chondrogenic potential compared to the wild-type protein. Limited proteolysis of the mutant recombinant proproteins resulted in a fragment pattern profoundly different from wild-type proGDF5. Modeling of a part of the GDF5 proregion into the known three-dimensional structure of TGF $\beta 1$ latency-associated peptide revealed that the homologous positions of both mutations are conserved regions that may be important for the folding of the mature protein or the assembly of dimeric protein complexes. We hypothesize that the missense mutations p.T201P and p.L263P interfere with the protein structure and thereby reduce the amount of fully processed, biologically active GDF5, finally causing the clinical loss of function phenotype.
\end{abstract}

(C) 2014 Elsevier Ltd. All rights reserved.

\section{Introduction}

Growth and differentiation factor 5 (GDF5) acts as a key player during skeletal development due to its ability to regulate cartilage formation. GDF5 is expressed in prechondrogenic aggregates and is an early marker for the joint forming regions. The promotion of cell assembly increases the size of these condensations. In later stages of cartilage formation, GDF5 also stimulates chondrocyte 
proliferation. Besides chondrogenesis, the growth factor plays a crucial role in the formation of tendons and ligaments [1,2].

GDF5 belongs to the TGF $\beta$ superfamily and shares a conserved structure and signal transduction mechanism with its family members. Full length (FL) GDF5 is translated as an inactive preproprotein. After the signal peptide is removed by processing in the Golgi apparatus, the proprotein is supposed to form a homodimer. Subsequently, the proregion is removed by prohormone convertases of the furin type at the processing site [RRKRR] N-terminal to position 382 presenting the start of the mature domain [3]. Cleavage is assumed to take part to a large extent in intracellular compartments. However, tissue-typedependent extracellular cleavage has been reported, and the proprotein and the mature growth factor can be secreted $[4,5]$. Mature GDF5 binds to the bone morphogenetic protein (BMP) type I receptor BMPR1A or BMPR1B, whereas the growth factor has a higher affinity to the latter one. The type I receptor dimer, in complex with BMP type II receptors, induces downstream signaling cascades, mainly via SMAD-dependent signal transduction (reviewed in Ref. [6]).

Although the GDF5 proregion comprises approximately $70 \%$ of the whole protein, its function is unknown. For the related proteins Activin $A$ and TGF $\beta 1$, it has been shown that secretion of the mature growth factors is impaired in the absence of the proregions [7]. The TGF $\beta 1$ proregion and the mature protein stay in a non-covalent complex that remains inactive since the latent TGF $\beta 1$ binding proteins are covalently bound to the proregion $[8,9]$. The proregions of the TGF $\beta$ superfamily are not highly conserved and functions differ between the individual family members.

The importance of the GDF5 proregion becomes apparent by missense mutations in this region leading to skeletal malformations. Examples are brachydactyly type A2 (p.R380Q [5]), brachydactyly type C (BDC) (p.M173V [10], p.L176P [11], p.S204R [12], p.R301stop [13]), Du Pan syndrome (p.R378Q [14]), chondrodysplasia Grebe type (p.Q100fs [15], p.L176P [11]) or proximal symphalangism (p.L373R [16]). Although some mutations in the proregion of GDF5 have been known for many years, only two reports elucidated potential disease-associated molecular mechanisms. One of these is $p . R 380 Q$, a missense mutation that causes brachydactyly type A2 and leads to impaired processing by prohormone convertases [5]. The second is the recently reported missense mutation p.L176P, which was found to cause BDC and chondrodysplasia Grebe type due to impaired GDF5 secretion [11].

Here, we present a cell biological and biochemical analysis of two missense mutations in the proregion of GDF5 causing BDC. We provide evidence that both mutations lead to a loss of function by reducing
GDF5 protein amount, possibly due to structural changes in the protein.

\section{Results}

\section{Heterozygosity for GDF5 mutations p.T201P and p.L263P causes a classical BDC phenotype}

We analyzed two families that were diagnosed with BDC [Online Mendelian Inheritance in Man $\AA$ (OMIM) \#113100]. Additionally observed skeletal manifestations in one of the families as described in Fig. 1a are most likely not associated features of BDC. Genetic analysis of the father and the two children of family 1 (Fig. 1a) revealed a heterozygosity for a missense mutation in the GDF5 gene at position c.601A > C (RefSeq: NM_000557.3), which is predicted to lead to the amino acid exchange of threonine to proline at position 201 (p.T201P). The mother was clinically inconspicuous. Analysis of the father and the child of family 2 (Fig. 1b) disclosed a heterozygosity for a missense mutation in the GDF5 gene at position c.788T > C (RefSeq: NM_000557.3) predicted to result in the amino acid exchange of leucine to proline at position 263 (p.L263P). The child (patient V) committed suicide at the age of 21 years. The mother was unaffected. Both identified mutation sites are located in the core domain of GDF5 (Fig. 2a) [17]. A sequence alignment of GDF5 between human, mouse, chicken, cow and zebrafish revealed that p.T201 is conserved among these species and that p.L263 is conserved among all shown species except for chicken, where leucine is replaced by valine, which is also a hydrophobic amino acid. A segment of the GDF5 proregion including the core domain was modeled into the known proTGF $\beta 1$ structure (Fig. $2 b$ ). The core domain of the GDF5 proregion corresponds to a part of the homologous arm domain of the proTGF $\beta 1$ protein, which together with the straitjacket segment encircles the mature TGF $\beta 1$ [18]. The homologous positions for T201 and L263 can be found in the $\beta 1$ and the $\beta 4$ strand of the TGF $\beta 1$ proregion, respectively.

\section{FL GDF5 ${ }^{\text {T201P }}$ and FL GDF5 ${ }^{\text {L263P }}$ lead to reduced BMPR1A and BMPR1B activation}

Mature GDF5 is known to bind to the receptor BMPR1A and its high-affinity receptor BMPR1B and thereby inducing SMAD-dependent signal transduction. A luciferase assay using the SMAD binding element (SBE) in the fibroblast cell line $\mathrm{NIH} / 3 \mathrm{~T} 3$ was performed to observe SMAD signaling stimulated by FL GDF5 ${ }^{\mathrm{WT}}$, FL GDF5 ${ }^{\text {T201P }}$ or FL GDF5 ${ }^{\mathrm{L} 263 \mathrm{P}}$ (Fig. 3). In comparison to cells transfected with a control vector, FL GDF5 ${ }^{\mathrm{WT}}$ led to an induction of luciferase activity when cotransfected with Bmpr1a or Bmpr1b 

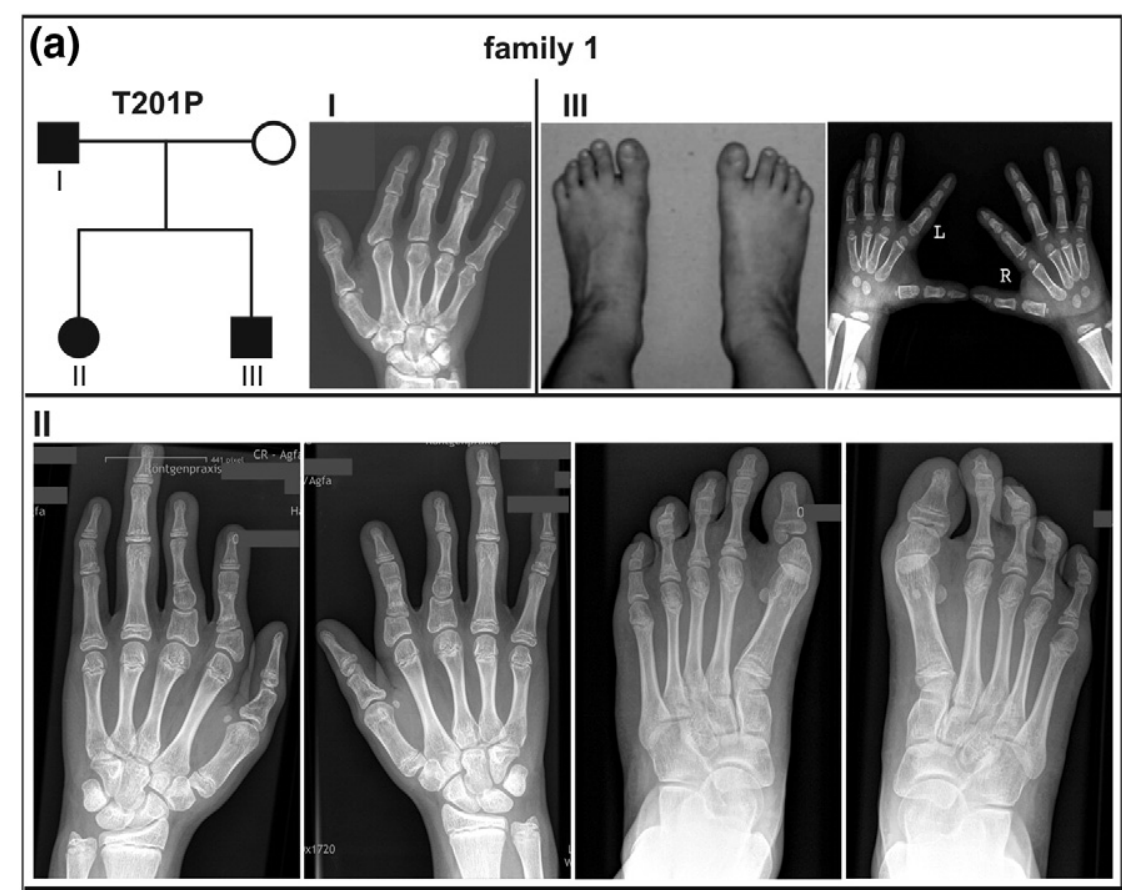

(b)

family 2
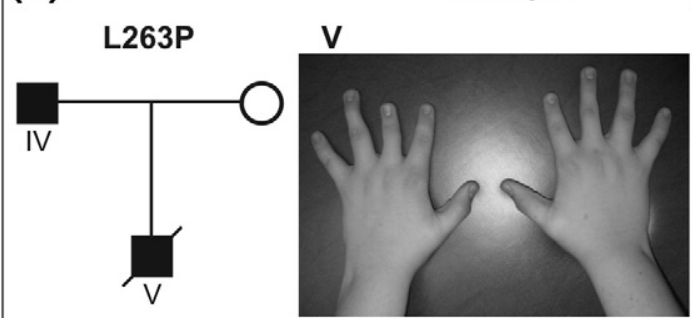

IV
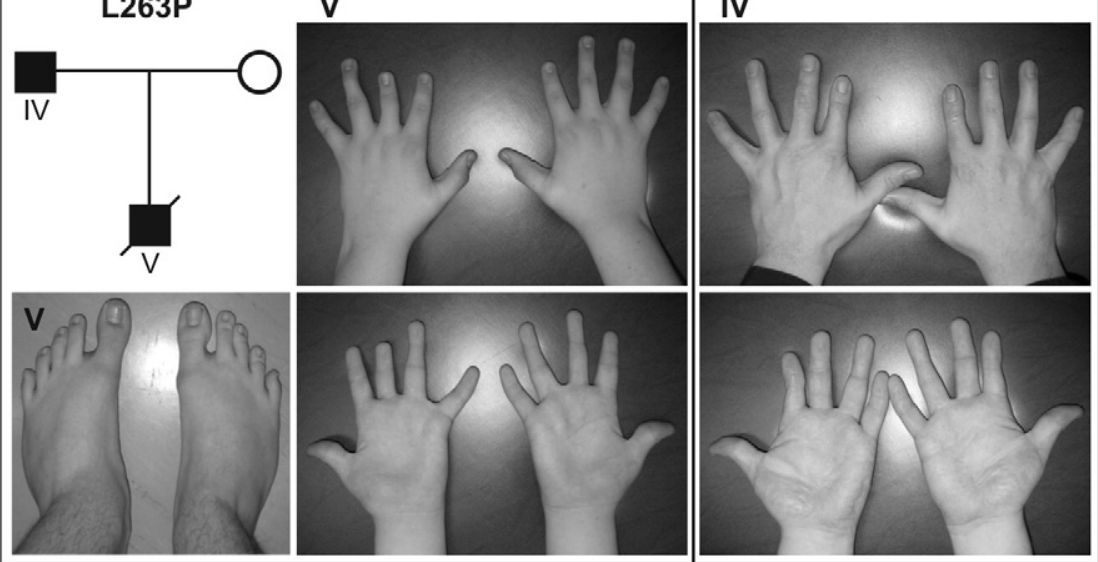

Fig. 1. Heterozygosity for missense mutations p.T201P and p.L263P causes dominant BDC. Affected family members of two pedigrees with different missense mutations in the proregion of GDF5 show typical features of BDC. (a) Three members of family 1 show heterozygosity for the missense mutation c.601A $>$ C predicted to lead to the amino acid exchange p.T201P (patients I-III). Patient I shows a mild phenotype with a shortened and laterally deviated index finger and a shortened middle finger. Patient II exhibits a more severe phenotype, showing shortened metacarpal and phalanges of the thumb, a hyperphalangy of the index and middle finger and shortened phalanges of the index, middle and little finger. The phalanges of the toes are shortened, and the great toe is laterally deviated. Patient III shows mild phenotypic features. The metacarpal and phalanges of the thumb are shortened and a clinodactyly of the little finger is present. The feet are not affected. (b) In family 2, the heterozygous missense mutation c.788T > C is predicted to lead to the amino acid exchange p.(L263P) (patients IV and V). Patient IV shows mild phenotypical features in the hand. The index and middle fingers are shortened and deviated to the left. The ring fingers appear distally narrowed. In addition, he has an unilateral congenital coax luxation, a bilateral pes planus and an hallux valgus that was operated at the age of 15 years. Patient $V$ shows typical BDC features such as a shortened index and middle finger. Additionally, an unilateral congenital coax luxation, a bilateral pes planus with talus vertivalis and a hallux varus were diagnosed. These skeletal manifestations in the father and the son are not typical for BDC and should be considered as independent. Patient $\mathrm{V}$ has committed suicide at the age of 21 years.

compared to the activation by the respective receptor. In contrast, FL GDF5 $5^{\text {T201P }}$ showed considerably less luciferase activity when cotransfected with either
Bmpr1a or Bmpr1b. FL GDF5 ${ }^{\mathrm{L} 263 \mathrm{P}}$ was not able to induce SMAD signaling upon Bmpr1a cotransfection whereas signaling via BMPR1B was slightly 

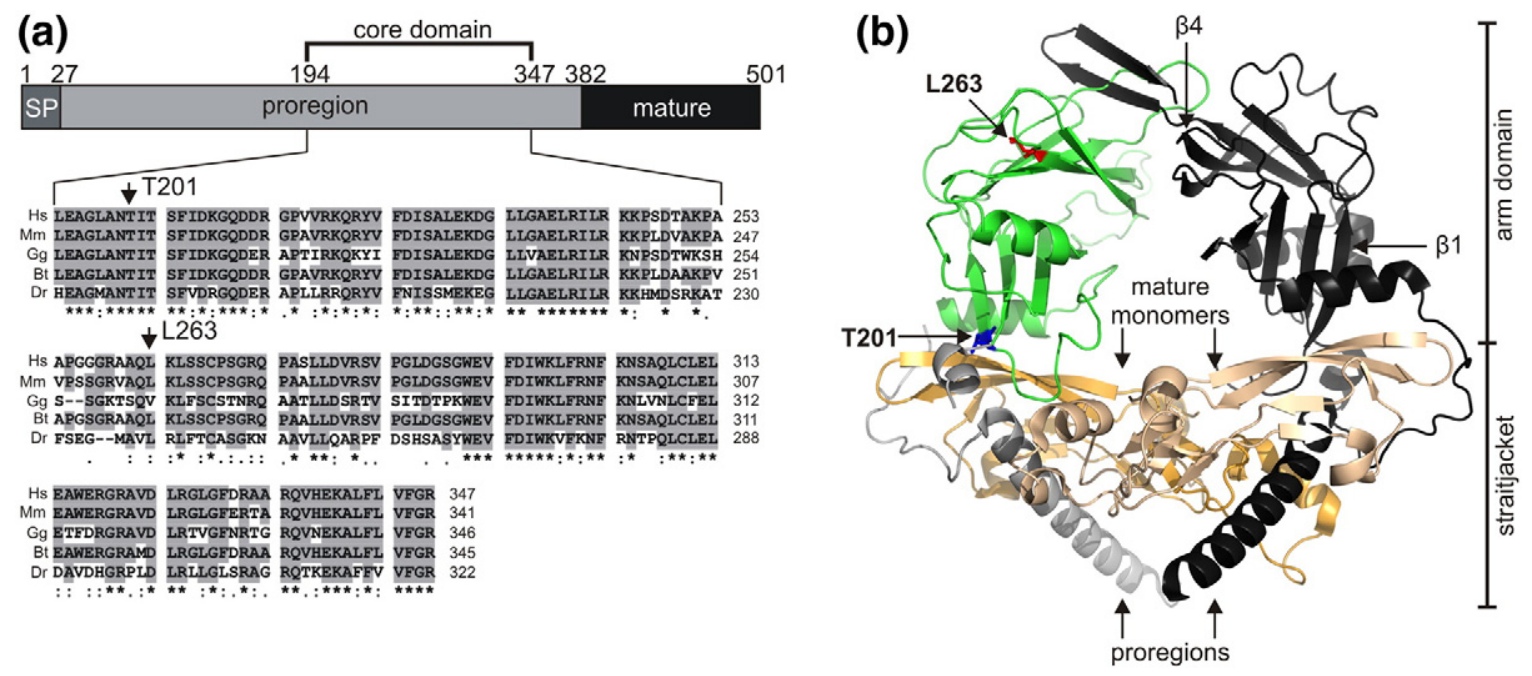

Fig. 2. p.T201 and p.L263 are located in the core domain of GDF5. (a) Scheme of preproGDF5 with sequence alignment of the previously identified core domain [17] within the proregion. The GDF5 preproprotein consists of the signal peptide (SP), the proregion and the mature domain. Protein sequences from human, mouse, chicken and cow GDF5 and contact, the zebrafish analogon of GDF5, were aligned (Hs, Homo sapiens; Mm, Mus musculus; Gg, Gallus gallus; Bt, Bos taurus; Dr, Danio rerio). Residues T201 and L263, which are replaced by proline in the patients, are located in the core domain, showing high conservation among different species. (b) A part of GDF5, including the core domain, was modeled into the structure of one proTGF $\beta 1$ monomer. The TGF $\beta 1$ proregions (shown in black and gray) clasp around the mature proteins (beige and orange). The modeled GDF5 structure is depicted in green and the positions T201 and L263 are highlighted (blue and red).

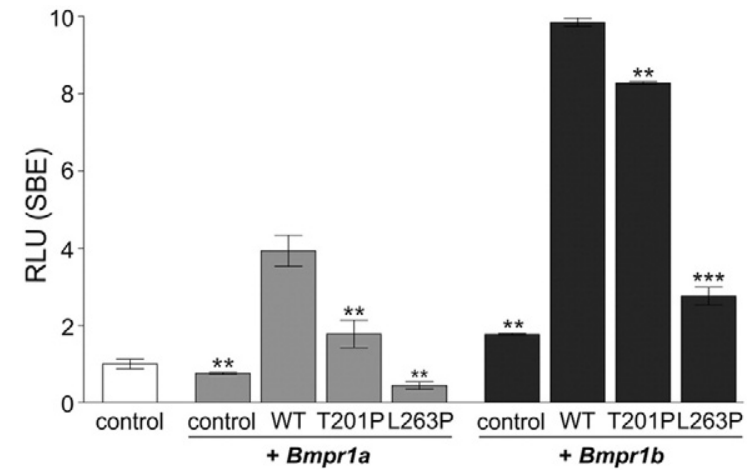

Fig. 3. FL GDF5 ${ }^{\mathrm{T} 201 \mathrm{P}}$ and FL GDF5 $5^{\mathrm{L} 263 \mathrm{P}}$ lead to reduced BMPR1A and BMPR1B activation. A control vector, $F L$ GDF5 ${ }^{W T}$, FL GDF5 ${ }^{T 201 P}$ or FL GDF5 $5^{L 263 P}$ was cotransfected with Bmpr1a or Bmpr1b in NIH/3T3 cells. Relative luciferase expression of the control vector (without receptor cotransfection) was set to 1.0. FL GDF5 ${ }^{\mathrm{WT}}$ shows considerable induction of SMAD signaling upon cotransfection of both receptors. In contrast, FL GDF5 ${ }^{\text {T201P }}$ shows reduced SMAD activation when cotransfected with Bmpr1a and Bmpr1b. FL GDF5 ${ }^{\text {L263P }}$ does not induce luciferase expression when cotransfected with Bmpr1a and severely reduces SMAD signaling after Bmpr1b cotransfection. All values are given as mean $\pm S D$ of samples from one representative of three experiments. Significant differences in comparison to $F L$ GDF5 ${ }^{W T}$ cotransfected with the respective receptor are given; two-tailed Student's $t$ test was performed $\left({ }^{* *} p<0.01\right.$, $\left.{ }^{* \star \star} p<0.001\right)$. increased but could not accomplish the level that was obtained with FL GDF5 ${ }^{\mathrm{WT}}$. These findings point to a diminished activity of FL GDF5 due to the investigated missense mutations affecting signaling via BMPR1A and the high-affinity receptor BMPR1B.

\section{FL GDF5 ${ }^{\text {T201P }}$ and FL GDF5 ${ }^{\text {L263P }}$ gave rise to less chondrogenic activity and SMAD signaling than FL GDF5 ${ }^{\text {WT }}$ due to reduced protein levels}

Since the induction of cartilage formation is a crucial function of GDF5, an in vitro assay for chondrogenesis was performed to elucidate functional changes of $\mathrm{FL}$ GDF5 $^{\text {T201P }}$ and FL GDF5 ${ }^{\mathrm{L} 263 \mathrm{P}}$. Cells were isolated from chicken limb buds, seeded at high cell densities and infected with an empty virus or a virus expressing FL GDF5 ${ }^{W T}$, FL GDF5 T201P or FL GDF5 ${ }^{L 263 P}$. The accumulation of cartilaginous matrix was monitored by Alcian blue staining (Fig. 4a). Whereas FL GDF5 ${ }^{\mathrm{WT}}$ was able to strongly induce differentiation of precursor cells, both mutant variants showed significantly lower chondrogenic potential. Thus, endogenous SMAD1/5/8 phosphorylation was monitored by performing a Western blot with micromass cells that were infected with an empty virus or a virus expressing $F L G D F 5^{W T}, F L G D F 5^{T 201 P}$ or $F L$ GDF5 ${ }^{L 263 P}$ (Fig. 4b). It was demonstrated that $F L$ $G D F 5^{W T}$ overexpression can strongly increase the 
(a)

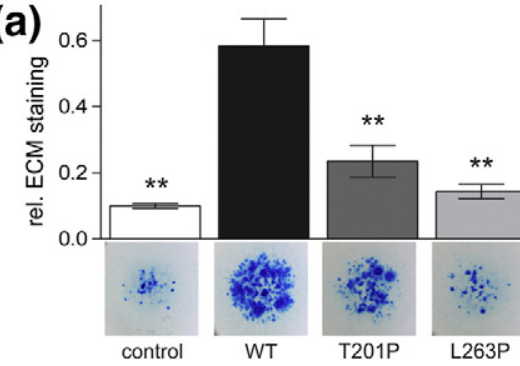

(b)

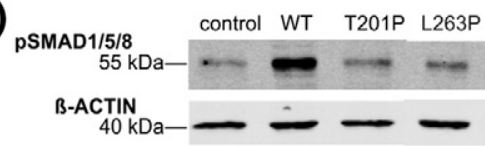

(c)

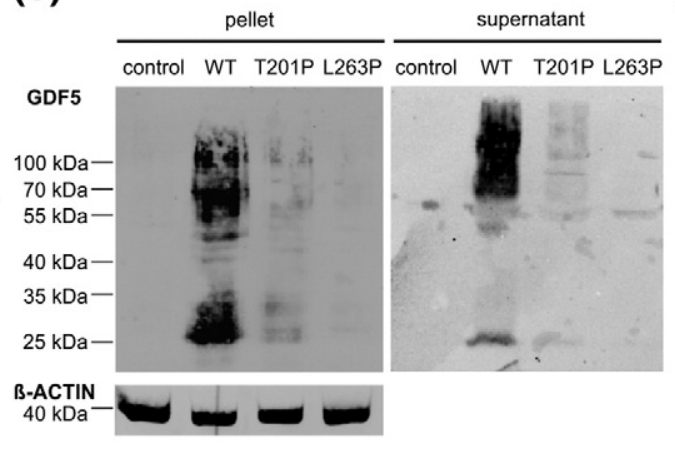

(d)

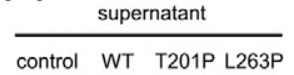

Fig. 4. FL GDF5 $5^{\mathrm{T} 201 \mathrm{P}}$ and FL GDF5 ${ }^{\mathrm{L} 263 \mathrm{P}}$ gave rise to less chondrogenic activity and SMAD signaling than FL GDF5 ${ }^{\mathrm{WT}}$ due to reduced protein levels. FL GDF5 ${ }^{W T}, F L G D F 5^{T 201 P}$ or $F L G D F 5^{L 263 P}$ was overexpressed in chicken micromass cultures using the RCAS virus. Empty vector overexpression serves as a control. (a) Alcian blue staining of cultures shows that FL GDF5 ${ }^{\mathrm{WT}}$ enhances chondrogenic differentiation of mesenchymal progenitor cells in vitro whereas both FL GDF5 variants show considerably less induction of chondrogenesis. All bars show mean \pm SD of triplicate samples from one representative of three experiments. Significant differences in comparison to FL GDF5 ${ }^{\mathrm{WT}}$ are given; two-tailed Student's $t$ test was performed $\left({ }^{* *} p<0.01\right)$. (b) Western blot of cell pellets of micromass cultures was performed using an antibody against phosphorylated SMAD1/5/8. $\beta$-ACTIN was used as a loading control. FL GDF5 ${ }^{\mathrm{WT}}$ considerably enhances phosphorylation of SMAD1/5/8 whereas mutant FL GDF5 variants do not induce SMAD1/5/8 activation. Samples were loaded on the same gel but were applied non-contiguously. (c) Western blot of cell pellets and supernatants of micromass cultures was performed using an anti GDF5 antibody. $\beta$-ACTIN was used for control. Upon FL GDF5 ${ }^{W T}$ overexpression, proprotein is detected in the pellet and the supernatant, mature GDF5 $(\sim 25 \mathrm{kDa})$, can be seen in both fractions. FL GDF5 ${ }^{\text {T201P }}$ and FL GDF5 ${ }^{\mathrm{L} 263 \mathrm{P}}$ led to a severe reduction of protein amount in the pellet and the supernatant and less or no mature protein can be detected. (d) Zinc staining was performed after loading of supernatants of micromass cultures onto a SDS gel. The total amount of protein was comparable for all samples.

protein level of pSMAD1/5/8 whereas both mutant FL GDF5 variants did not result in the induction of SMAD1/5/8 phosphorylation compared to the control. To investigate whether the reduced signaling activity of the FL GDF5 variants would be caused by differences in the protein amounts, we tested the expression of wild-type and mutant FL GDF5 in a Western blot of cell lysates and supernatants using an anti GDF5 antibody (Fig. 4c). Zinc staining of a gel loaded with samples of the supernatants was performed to demonstrate that equal total protein amounts were used (Fig. 4d). Upon overexpression of $F L G D F 5^{W T}$, proforms and mature protein could be detected in the cell pellets and medium. When $F L$ GDF5 ${ }^{\text {T201P }}$ was overexpressed, considerably less protein could be observed in the cell pellets and in the medium. No GDF5 was present upon overexpression of $F L G D F 5^{L 263 P}$. These results indicate that the amino acid exchanges T201P and L263P cause reduced protein levels of GDF5 and decrease GDF5 induced SMAD signaling.

\section{Recombinant human proGDF5 ${ }^{\mathrm{T} 201 \mathrm{P}}$ and proGDF5 ${ }^{\mathrm{L263P}}$ have a decreased chondrogenic potential}

To test whether the reduced activity of $F$ GDF5 $^{\text {T201P }}$ and FL GDF5 ${ }^{\text {L263P }}$ would be caused by the diminished protein levels or result from structural changes, we used recombinant human (rh) proproteins in a chondrogenesis assay, which further allowed us to examine their effect dose dependently. Production of cartilaginous matrix induced by recombinant proteins was monitored by Alcian blue staining. All three tested proteins behaved in a dose-dependent manner. Although identical protein concentration series were used in this assay, rh proGDF5 ${ }^{\mathrm{WT}}$ showed significantly higher chondrogenic activity than rh proGDF5 $^{\text {T201P }}$ and rh proGDF5 ${ }^{\mathrm{L} 263 \mathrm{P}}$ (Fig. 5). The reduced potential of both rh proGDF5 variants to induce the differentiation of progenitor cells into the chondrogenic lineage provides additional evidence for the assumed loss of function caused by the missense mutations. We tested if the reduced activities are due to an altered maturation of GDF5 by proprotein convertases and performed a SDS-PAGE analysis of the rh proGDF5 variants digested with furin. No difference was seen between GDF5 ${ }^{\mathrm{WT}}$ and GDF5 ${ }^{\mathrm{T} 201 \mathrm{P}}$ or GDF5 $^{\mathrm{L263P}}$ (Supplemental Fig. S1). These results led to the hypothesis that the investigated GDF5 variants may lead to other structural differences in the protein.

\section{Limited proteolysis leads to abnormal proteolysis products for rh proGDF5 ${ }^{\text {T201P }}$ and rh proGDF5 ${ }^{\mathrm{L} 263 \mathrm{P}}$}

Limited proteolysis assay with proforms of GDF5 $^{\mathrm{WT}}$ and the two mutants using trypsin was used as a simple biochemical method to obtain 
(a)

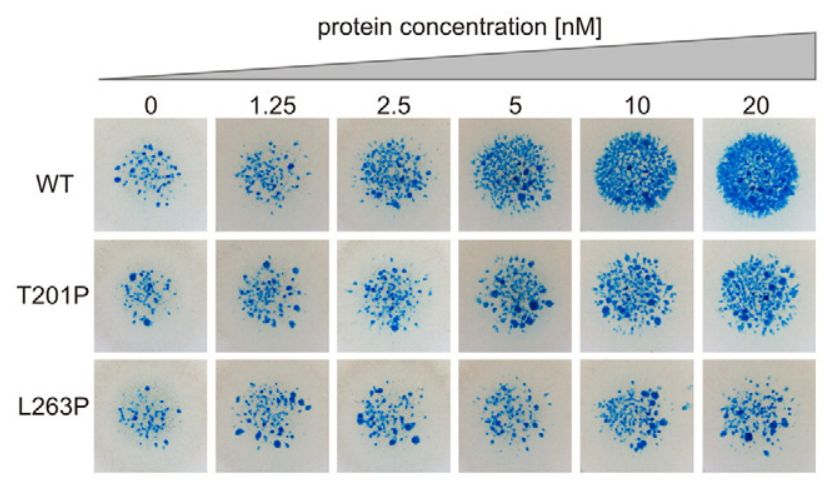

(b)

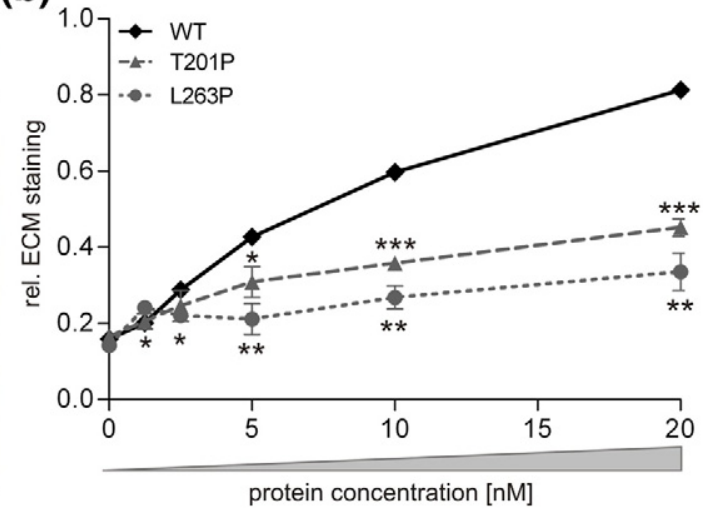

Fig. 5. Chondrogenic activity of rh proGDF5 is diminished by the mutations p.T201P and p.L263P. Recombinant proproteins of GDF5 $^{\mathrm{WT}}$, GDF5 ${ }^{\mathrm{T} 201 \mathrm{P}}$ and GDF5 ${ }^{\mathrm{L} 263 \mathrm{P}}$ were used in a chondrogenesis assay to stimulate differentiation of micromass cultures at the indicated concentrations. Cells were seeded at high densities and stimulated with the proteins for comparison of rh proGDF5 ${ }^{\mathrm{WT}}$ and variants. (a) Alcian blue staining of extracellular matrix displays induction of chondrogenesis. Rh proGDF5 ${ }^{\mathrm{T} 201 \mathrm{P}}$ and $\mathrm{rh}$ proGDF5 ${ }^{\mathrm{L} 263 \mathrm{P}}$ gave rise to lower chondrogenesis than rh proGDF5 ${ }^{\mathrm{WT}}$. (b) Quantification of Alcian blue staining demonstrates reduced chondrogenic potential of mutant rh proGDF5 variants. All values are given as mean \pm SD of triplicate samples from one representative of three experiments. Significant differences in comparison to the respective concentration of rh proGDF5 ${ }^{\mathrm{WT}}$ are given; two-tailed Student's $t$ test was performed $\left({ }^{*} p<0.05,{ }^{* *} p<0.01,{ }^{* * *} p<0.001\right)$.

information regarding protein structure and conformational changes of the different GDF5 variants. Trypsin cleaves peptide bonds C-terminal to lysine and arginine, provided that they are surface exposed and not integrated into a rigid structure $[19,20]$. Therefore, it is a classical approach to detect conformational differences [21]. Previous results obtained with the proforms of NGF and BMP2 had shown that trypsin can be used to convert proforms into mature forms $[22,23]$. At a mass ratio of trypsin toward proform of 1:1000 to 1:50, a band of ca $25 \mathrm{kDa}$ was visible in the proteolysis products of rh proGDF5 ${ }^{\mathrm{WT}}$ (Fig. 6). This band corresponded in size to mature GDF5 that was loaded as a control. Finally, a proteaseresistant product migrating at ca $20 \mathrm{kDa}$ was detectable in case of rh proGDF5 ${ }^{\mathrm{WT}}$ and rh proGDF5 ${ }^{\mathrm{T} 201 \mathrm{P}}$. This band probably corresponds to a truncated form of mature GDF5 as it did not arise when the separate proregions were subjected to limited proteolysis (data not shown). The 17-kDa core fragment of $\mathrm{rh}$ proGDF5 ${ }^{\mathrm{T} 201 \mathrm{P}}$, in contrast to $\mathrm{rh}$ proGDF5 ${ }^{\mathrm{WT}}$, was digested at a mass ratio of trypsin toward proform of $1: 10$. More pronounced differences in the proteolysis pattern were obtained with rh proGDF5 ${ }^{\mathrm{L263P}}$. Here, already at low protease concentrations, a smear was visible. Furthermore, the $17-\mathrm{kDa}$ core domain that was clearly discernible for rh proGDF5 ${ }^{\mathrm{WT}}$ and $\mathrm{rh}$ proGDF5 ${ }^{\text {T201P }}$ was not seen.

\section{Discussion}

In this study, we analyzed two novel heterozygous missense mutations in the proregion of GDF5 associated with BDC: p.T201P and p.L263P. The patients show shortened fingers or toes, hyperphalangy or clinodactyly that are classical disease manifestations for BDC. BDC showed intrafamilial and interfamilial clinical variability and was inherited in a dominant pattern and co-segregated with the investigated GDF5 missense mutations. The dominant pattern of inheritance is typical for BDC, whereas homozygous loss of GDF5 leads to more severe phenotypes, like acromesomelic chondrodysplasia (OMIM \#201250) or chondrodysplasia Grebe type (OMIM \#200700). In these latter diseases, the phenotype is more pronounced, not only affecting the hands and feet but also leading to severe shortening of the forearms and forelegs. It seems that the digits are most sensitive to reduced GDF5 function as skeletal elements already show pronounced phenotypic manifestations upon heterozygous loss of function of GDF5, like in BDC.

GDF5 ${ }^{\text {T201P }}$ and GDF5 ${ }^{\mathrm{L} 263 \mathrm{P}}$ were tested in functional assays regarding their chondrogenic potential and signal transduction. On a molecular level, the signal transduction of GDF5 is mainly mediated through the SMAD-dependent pathway via phosphorylation of SMAD1/5/8 by its receptors BMPR1A and BMPR1B. The activation of the SMAD pathway was reduced by both FL GDF5 variants in a luciferase assay using a SMAD-specific reporter in fibroblasts affecting both receptors. Moreover, the protein levels of pSMAD1/5/8 were considerably lower in primary cells that expressed either FL GDF5 ${ }^{T 201 P}$ or FL GDF5 ${ }^{L 263 P}$ compared to $F L G D F 5^{W T}$ overexpressing cells.

The missense mutations p.T201P and p.L263P were found to be heterozygous in the patients, 


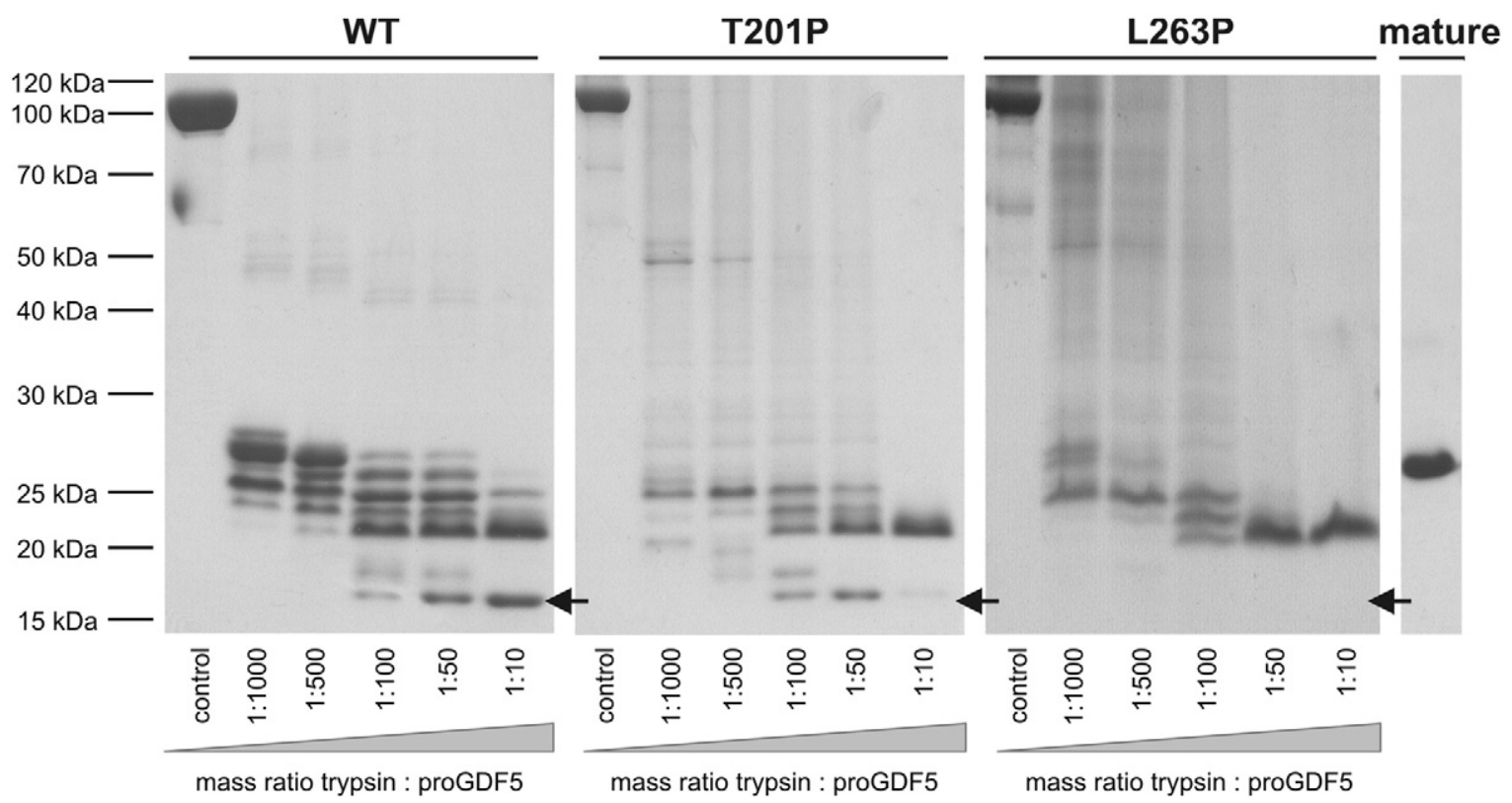

Fig. 6. Limited proteolysis with trypsin reveals that rh proGDF5 ${ }^{\mathrm{T} 201 \mathrm{P}}$ and $\mathrm{rh}$ proGDF5 $5^{\mathrm{L} 263 \mathrm{P}}$ induce differences at the structural level. Upon limited proteolysis with trypsin with the given ratios, proteolysis products were analyzed by SDS-PAGE. The occurrence of different proteolysis products under otherwise identical conditions and concentrations of trypsin implicates that the two exchanges lead to structures that are divergent from rh proGDF5 ${ }^{\mathrm{WT}}$. In contrast to rh proGDF5 $^{\mathrm{WT}}$, the core fragment (arrow) of rh proGDF5 ${ }^{\mathrm{T201P}}$ is more sensitive to trypsin. After digestion of rh proGDF5 ${ }^{\mathrm{L263P}}$, the 17-kDa core fragment cannot be detected.

meaning that one functional wild-type allele and one allele carrying a mutation is present. As GDF5 is known to form dimers, there are three combinations of monomers possible: $25 \%$ of the GDF5 dimers consist of two wild-type monomers, which will show normal activity. Another $25 \%$ consist of two mutant monomers, which will lead to impaired functionality. Moreover, $50 \%$ of the GDF5 dimers can be formed of a wild-type monomer and a mutant monomer. Therefore, mutant GDF5 might impair the function of the wild-type allele as well. Although this aspect was not investigated here, it was shown in another study that the proregion mutation FL GDF5 ${ }^{\mathrm{L} 176 \mathrm{P}}$ had a dominant negative effect on the secretion of $F L$ GDF5 ${ }^{\mathrm{WT}}$ that was co-expressed with $L T B P$ [11]. As a consequence, FL GDF5 ${ }^{\mathrm{L} 176 \mathrm{P}}$ showed no induction of alkaline phosphatase activity, a marker for chondrogenesis. The amounts of GDF5 proproteins in the cell pellets were comparable for GDF5 ${ }^{\text {WT }}$ and GDF5 ${ }^{\mathrm{L} 176 \mathrm{P}}$.

In contrast, our Western blot analysis of cells overexpressing FL GDF5 $5^{T 201 P}$ or FL GDF5 $5^{L 263 P}$ revealed that less proprotein and less mature GDF5 were seen. This correlates with the reduced SMAD signaling activity and the accordingly decelerated chondrogenic differentiation induced by both $F L$ GDF5 variants after ectopic expression.

There are three possible explanations for the reduced protein levels of the GDF5 variants: (1) Mutant GDF5 is not efficiently translated. (2) The translated
GDF5 variants are not correctly folded and therefore not detected by the antibody. (3) The GDF5 variants are preferentially degraded by the protein quality control machinery. Surprisingly, if protein levels were adjusted by using recombinant proteins, both mutant proteins still led to significantly less accumulation of chondrogenic matrix by micromass cells, arguing that the FL GDF5 variants, even when expressed and secreted to same levels, show less biological activity than FL GDF5 ${ }^{\mathrm{WT}}$.

Moreover, the mutations p.T201P and p.L263P led to an increased sensitivity to protease digestion of the two mutant proproteins in comparison to rh proGDF5 ${ }^{\mathrm{WT}}$ as it was shown by limited trypsinolysis. This is supported by the absence of the 17-kDa core domain after proteolysis of rh proGDF5 ${ }^{\mathrm{L} 263 \mathrm{P}}$, which had been shown earlier to constitute a subdomain within the BMP2 proregion. The analysis suggests that the amino acid exchanges modify protein structure. Incorrect folding may lead to a diminished protein stability that enforces the protein degradation process. The three-dimensional structure of the GDF5 proprotein has not been solved yet, but the structure of proTGF $\beta 1$ has been published [18]. A comparison of proTGF $\beta 1$ to sequences of several other BMPs, including GDF5, revealed that only some regions of these growth factors are highly conserved, but they might share a similar basic structure as all proregions seem to have a similar fold [18]. The proregion of TGF $\beta 1$ contains an arm domain that associates with 
the arm domain of another proTGF $\beta 1$ protein to a homodimer and encircles the mature protein together with the proregion straitjacket region [18]. According to the homology model, both tested GDF5 mutations are located in the arm domain. The homologous positions for the GDF5 variants are found in regions of proTGF $\beta 1$ that are probably important for the structural integrity of the protein. The corresponding position of GDF5 ${ }^{\mathrm{T} 201}$ localizes within the $\beta 1$ strand that serves as a critical region for templating the folding of the mature domain [18]. Moreover, the mature protein facing end of the $\beta 1$ strand of TGF $\beta 1$ blocks the binding to type I receptors. The homologous position of GDF5 ${ }^{\mathrm{L} 263}$ can be found in the $\beta 4$ strand, whose sequence seems to be variable between different growth factors as it lacks conserved residues [18]. The $\beta 4$ strand contains the interface between two arm domains and is therefore necessary for protein dimerization. It seems likely that amino acid exchanges within these strands can damage the structure of the protein. Nevertheless, a threedimensional structure of the GDF5 proprotein is needed to better assess alterations in the protein structure due to mutations. The sequences of TGF $\beta 1$ and GDF5 proregions are not highly conserved, and therefore, their structures might differ from each other.

In summary, the newly described amino acid exchanges p.T201P and p.L263P reduce the protein amount and lead to a decreased biological availability of the growth factor GDF5. These data support the hypothesis that the proregion of GDF5, similar to TGF $\beta 1$ and Activin, plays an essential role in the biogenesis of GDF5. The investigated mutations probably interfere with this function.

\section{Materials and Methods}

\section{Clinical investigation and molecular analysis}

All clinical investigations were conducted in accordance with the provision of the Declaration of Helsinki principles. Diagnosis of BDC was based on clinical investigation and genetic analyses were performed. Informed consent was obtained from all persons or their legal guardians for underage persons. Genomic DNA was purified from blood and mutation screening in GDF5 performed as previously described [10].

\section{Sequence alignment and structure modeling}

Sequences from human, mouse, chicken and cow GDF5 and contact, the zebrafish analogon of GDF5, were aligned using ClustalW $W^{\dagger}$.

The structure of TGF $\beta 1$ including its proregion \{Protein Data Bank (PDB) ID: 3RJR [18]\} was used as a template to model the three-dimensional proGDF5 structure from position E171-R347 using SWISS-MODEL * [24-26]. The sequence segment comprises the GDF5 core domain [17].
The corresponding TGF $\beta 1$ segment (E46-T234) was replaced by the modeled GDF5 structure using PyMOL within the TGF $\beta 1$ protein (PDB ID: 3RJR) [27].

\section{Cloning of constructs and virus production}

The missense mutations coding for T201P or L263P were inserted into the coding sequence of human GDF5 in the shuttle vector pSLAX13 via site-directed in vitro mutagenesis (primers: T201P forward 5'-GGAGGCTGGCCTGGCCAACCCCATCACCAGCTTTATTGAC-3', T201P reverse 5'-GTCAATAAAGCTGGTGATGGGGTTGGCCAGGCCAGCCTCC-3', L263P forward 5'- AGGCGGGCGGGCTG CCCAGCCGAAGCTGTCCAGCTGCCCCAG-3', L263P reverse 5'-CTGGGGCAGCTGGACAGCTTCGGCTGGGCAGCCCGCCCGCCT-3') and confirmed by sequencing. The inserts were subcloned into the viral vector RCAS(BP)A after Clal restriction. The plasmids were transfected into DF1 cells for virus production and the harvested medium containing secreted viruses was concentrated as described previously to enhance the virus titer [28]. For titer determination, plaque-forming units (PFU) (PFU/ml) were quantified.

\section{Luciferase assay}

FL GDF5 $5^{\mathrm{WT}}, F L$ GDF5 $5^{\mathrm{T} 201 \mathrm{P}}$ and FL GDF5 $5^{\mathrm{L} 263 \mathrm{P}}$ sequences were subcloned from pSLAX13 into the expression vector pCS2+ after restriction with Clal. Sequences of mouse Bmpr1a and Bmpr1b were inserted into the shuttle vector PSLAX13 and subcloned into the expression vector pCS2 + after Clal restriction.

$\mathrm{NIH} / 3 \mathrm{~T} 3$ cells (American Type Culture Collection) were seeded into a 96 -well plate at a density of $1 \times 10^{4}$ cells/well and cultured in growth medium [Dulbecco's modified Eagle's medium (DMEM) high glucose (Lonza), 10\% fetal bovine serum (FBS) superior (Biochrom), $2 \mathrm{mM}$ L-glutamine (Lonza), pen/strep (Lonza)] for $24 \mathrm{~h}$ at $37{ }^{\circ} \mathrm{C}$ and $5 \% \mathrm{CO}_{2}$. Cells were transfected with the firefly luciferase reporter pGL3ti-SBE [29] and the Renilla luciferase normalization vector $\mathrm{pRL}$-TK (Promega) and the empty vector $\mathrm{pCS} 2+$, expression constructs of $F L$ GDF5 $5^{W T}, F L$ GDF5 ${ }^{T 201 P}, F L$ $G D F 5^{L 263 P}$, Bmpr1a and Bmpr1b as indicated. For transfection, Lipofectamine 2000 (Life Technologies) was used following the manufacturer's protocol. Medium was changed to $1 \%$ FBS upon transfection. Forty-two hours after transfection, cells were lysed in potassium phosphate buffer (9 mM potassium dihydrogen phosphate, $91 \mathrm{mM}$ dipotassium phosphate, $0.2 \%$ Triton X-100) and luciferase activity was determined using the Mithras LB 940 (Berthold Detection Systems [30]). Relative luciferase activity of the control vector (without receptor cotransfection) was set to 1.0 for graphical presentation.

\section{Chicken micromass culture}

Fertilized chicken eggs (Valo Biomedia) were incubated for 4.5 days until Hamburger Hamilton stage 24-25. The limb buds of the embryos were dissected and digested to achieve a single cell suspension [5]. Cells were seeded as drop cultures in 24-well plates and incubated in growth medium [DMEM:F12 (Biochrom), 10\% FBS superior (Biochrom), 0.2\% chicken serum (Sigma), 2 mM L-glutamine (Lonza), $100 \mathrm{U} / \mathrm{ml} \mathrm{pen/strep} \mathrm{(Lonza)]} \mathrm{at} 39{ }^{\circ} \mathrm{C}$ and $5 \% \mathrm{CO}_{2}$. 
For ectopic expression, micromass cultures were infected with an empty virus control or a virus carrying either wild-type or mutant FL GDF5 using a titer of $0.5 \times 10^{7} \mathrm{PFU} / \mathrm{ml}$.

To stimulate cartilage formation in micromass cultures, we added the recombinant proprotein GDF5 ${ }^{\mathrm{WT}}$, GDF5 ${ }^{\mathrm{T} 201 \mathrm{P}}$ or $\mathrm{GDF}^{\mathrm{L} 263 \mathrm{P}} 24 \mathrm{~h}$ after seeding of the cells at the indicated final concentrations.

Five days after viral infection or 3 days after stimulation with recombinant proteins, cultures were fixed with Kahle's fixative $(28.9 \%$ ethanol, $0.37 \%$ formaldehyde, $3.9 \%$ acetic acid) and stained with $0.05 \%$ Alcian blue (Sigma-Aldrich, dissolved in $0.1 \mathrm{M} \mathrm{HCl}$ ) that incorporates into the extracellular matrix of chondrocytes. Photo documentation was performed after drying of the cultures. For quantification, the dye was extracted with $6 \mathrm{M}$ guanidine hydrochloride and absorption was measured photometrically at $595 \mathrm{~nm}$.

\section{Western blot}

Chicken micromass cultures were infected with an empty virus or a virus expressing $F L$ GDF5 ${ }^{W T}$, FL GDF5 $5^{T 201 P}$ or FL $G D F 5^{L 263 P}$ as described in the previous section using a virus titer of $1 \times 10^{7} \mathrm{PFU} / \mathrm{ml}$.

Collected medium was precipitated after adding acetone. The cell pellets were lysed $(50 \mathrm{mM}$ Hepes, $10 \mathrm{mM}$ ethylenediaminetetraacetic acid, $10 \%$ glycerol, $1 \%$ Triton X-100, 50 mM NaCl, $1 \times$ Complete Mini, 1 mM PMSF, 2 mM $\mathrm{NaF}, 5 \mathrm{mM} \mathrm{Na}_{4} \mathrm{O}_{7} \mathrm{P}_{2}$ ) and homogenized using the TissueLyserll (Qiagen). Protein concentrations in cell pellets were determined with a BCA protein assay kit (Thermo Scientific). Samples were loaded onto a $12 \%$ SDS gel and blotted using the PerfectBlue Semi-Dry blot system (Peqlab), and the membrane was incubated in blocking buffer. Primary antibodies were allowed to bind to the membrane overnight. Secondary antibody was coupled to an infrared dye and membranes were scanned with the Odyssey Infrared Imaging System (LI-COR).

For detection of GDF5, the medium of micromass cultures was replaced by $1 \mathrm{ml}$ serum-free medium [DMEM:F12 (Biochrom), 2 mM L-glutamine (Lonza), $100 \mathrm{U} / \mathrm{ml}$ pen/strep (Lonza)] 6 days after infection. Twenty-four hours later, medium was collected. The antibody aMP5 (Biopharm) recognizes GDF5 under non-reducing conditions. For detection of pSMAD1/5/8 (antibody \#9511; Cell Signaling), cell pellets were collected 3 days after infection and the Western blot was performed under reducing conditions. For detection of $\beta$-ACTIN (antibody \#A2066 and antibody \#A5441; Sigma-Aldrich), cell extracts that were used for GDF5 or pSMAD1/5/8 detection were loaded in reducing sample buffer.

To demonstrate loading of equal amounts of total protein for the supernatant samples, we performed zinc staining using the reversible zinc stain kit (G-Biosciences) following the manufacturer's instructions.

\section{Production of recombinant proteins}

The coding sequences of proGDF5 ${ }^{W T}$, proGDF5 ${ }^{\text {T201P }}$ and proGDF5 ${ }^{L 263 P}$ lacking the signal peptide were subcloned into $\mathrm{pET} 15 \mathrm{~b}$. After recombinant expression in Escherichia coli BL21 (DE3), proteins were prepared from inclusion bodies of the harvested cells. The proteins were renaturated and subsequently purified via Heparin Sepharose chromatography (T. P. Thieme, unpublished results).

\section{Limited proteolysis with recombinant proteins}

For limited proteolysis, proteins at concentrations of $8 \mu \mathrm{M}$ were incubated with trypsin in $50 \mu \mathrm{l}$ of $0.1 \mathrm{M} \mathrm{Tris}-\mathrm{Cl}$ and $5 \mathrm{mM}$ ethylenediaminetetraacetic acid $(\mathrm{pH} \mathrm{8.5)}$ at ambient temperature for $30 \mathrm{~min}$. Mass ratios of trypsin to substrate protein are indicated in the figure legend. Proteolysis was stopped by aprotinin in a 5-fold molar excess over trypsin and by addition of SDS sample buffer. Immediately after proteolysis, products were analyzed by SDS-PAGE and subsequent Coomassie staining

\section{Accession numbers}

The description of BDC is deposited in the OMIM database under accession number 113100. The National Center for Biotechnology Information Reference Sequence NM_000557.3 was used for the GDF5 gene and the TGF $\beta 1$ proprotein structure is found in the Research Collaboratory for Structural Bioinformatics PDB under the accession number 3RJR.

Supplementary data to this article can be found online at http://dx.doi.org/10.1016/j.jmb.2014.07.029.

\section{Acknowledgements}

We acknowledge the excellent technical assistance of Mareen Schmidt-von Kegler and Gundula Leschik. Biopharm kindly provided the aMP5 antibody and we would like to thank Peter ten Dijke for making the plasmid pGL3ti-SBE available to us. Contributions were made possible by Deutsche Forschungsgemeinschaft funding through the BerlinBrandenburg School for Regenerative Therapies GSC 203 and a doctoral fellowship by the Sonnenfeld Stiftung to K.S. The work was funded by the Federal Ministry of Education and Research within the ProNet-T3-Protein-Kompetentnetzwerk-Halle: tools, targets, therapeutics and subproject Th04 to E.S.

Conflict of Interest Statement: All authors declare that there is no conflict of interest.

Received 28 March 2014; Received in revised form 10 July 2014; Accepted 24 July 2014 Available online 1 August 2014

Keywords: BMP; chondrogenesis; skeletal diseases; proprotein; chicken micromass 
†http://www.ch.embnet.org/software/ClustalW.html thttp://swissmodel.expasy.org/

Abbreviations used: GDF5, growth and differentiation factor 5; BMP, bone morphogenetic protein; BDC, brachydactyly type C; FL, full length; rh, recombinant human; SBE, SMAD binding element; PFU, plaque-forming units; DMEM, Dulbecco's modified Eagle's medium; FBS, fetal bovine serum; OMIM, Online Mendelian Inheritance in Man $尺$; PDB, Protein Data Bank.

\section{References}

[1] Francis-West PH, Abdelfattah A, Chen P, Allen C, Parish J, Ladher R, et al. Mechanisms of GDF-5 action during skeletal development. Development 1999;126:1305-15.

[2] Merino R, Macias D, Ganan Y, Economides AN, Wang X, Wu Q, et al. Expression and function of Gdf-5 during digit skeletogenesis in the embryonic chick leg bud. Dev Biol 1999;206:33-45.

[3] Luyten FP. Cartilage-derived morphogenetic protein-1. Int J Biochem Cell Biol 1997;29:1241-4.

[4] Anderson SB, Goldberg AL, Whitman M. Identification of a novel pool of extracellular pro-myostatin in skeletal muscle. $J$ Biol Chem 2008;283:7027-35.

[5] Ploger F, Seemann P, Schmidt-von Kegler M, Lehmann K, Seidel J, Kjaer KW, et al. Brachydactyly type A2 associated with a defect in proGDF5 processing. Hum Mol Genet 2008; 17:1222-33.

[6] Miyazono K, Kamiya Y, Morikawa M. Bone morphogenetic protein receptors and signal transduction. J Biochem 2010; 147:35-51.

[7] Gray AM, Mason AJ. Requirement for activin A and transforming growth factor-beta 1 pro-regions in homodimer assembly. Science 1990;247:1328-30.

[8] Miyazono K, Hellman U, Wernstedt C, Heldin CH. Latent high molecular weight complex of transforming growth factor beta 1. Purification from human platelets and structural characterization. J Biol Chem 1988;263:6407-15.

[9] Wakefield LM, Smith DM, Flanders KC, Sporn MB. Latent transforming growth factor-beta from human platelets. A high molecular weight complex containing precursor sequences. J Biol Chem 1988;263:7646-54.

[10] Schwabe GC, Turkmen S, Leschik G, Palanduz S, Stover B, Goecke TO, et al. Brachydactyly type C caused by a homozygous missense mutation in the prodomain of CDMP1. Am J Med Genet A 2004;124A:356-63.

[11] Farooq M, Nakai H, Fujimoto A, Fujikawa H, Kjaer KW, Baig $\mathrm{SM}$, et al. Characterization of a novel missense mutation in the prodomain of GDF5, which underlies brachydactyly type $\mathrm{C}$ and mild Grebe type chondrodysplasia in a large Pakistani family. Hum Genet 2013;132:1253-64.

[12] Everman DB, Bartels CF, Yang Y, Yanamandra N, Goodman FR, Mendoza-Londono JR, et al. The mutational spectrum of brachydactyly type C. Am J Med Genet 2002;112:291-6.

[13] Polinkovsky A, Robin NH, Thomas JT, Irons M, Lynn A, Goodman FR, et al. Mutations in CDMP1 cause autosomal dominant brachydactyly type C. Nat Genet 1997;17:18-9.

[14] Douzgou S, Lehmann K, Mingarelli R, Mundlos S, Dallapiccola B. Compound heterozygosity for GDF5 in Du Pan type chondrodysplasia. Am J Med Genet A 2008;146A: 2116-21.
[15] Faiyaz-Ul-Haque M, Ahmad W, Wahab A, Haque S, Azim $\mathrm{AC}$, Zaidi $\mathrm{SH}$, et al. Frameshift mutation in the cartilagederived morphogenetic protein 1 (CDMP1) gene and severe acromesomelic chondrodysplasia resembling Grebe-type chondrodysplasia. Am J Med Genet 2002;111:31-7.

[16] Yang W, Cao L, Liu W, Jiang L, Sun M, Zhang D, et al. Novel point mutations in GDF5 associated with two distinct limb malformations in Chinese: brachydactyly type $\mathrm{C}$ and proximal symphalangism. J Hum Genet 2008;53:368-74.

[17] Kuhfahl S, Hauburger A, Thieme T, Groppe J, Ihling C, Tomic S, et al. Identification of a core domain within the proregion of bone morphogenetic proteins that interacts with the dimeric, mature domain. Biochem Biophys Res Commun 2011;408:300-5.

[18] Shi M, Zhu J, Wang R, Chen X, Mi L, Walz T, et al. Latent TGF-beta structure and activation. Nature 2011;474:343-9.

[19] Rudolph R. The use of limited proteolysis in studies on protein folding and association. Biochem Soc Trans 1985;13:308-11.

[20] Zettlmeissl G, Rudolph R, Jaenicke R. Limited proteolysis as a tool to study the kinetics of protein folding: conformational rearrangements in acid-dissociated lactic dehydrogenase as determined by pepsin digestion. Arch Biochem Biophys 1983;224:161-8.

[21] Hubbard SJ. The structural aspects of limited proteolysis of native proteins. Biochim Biophys Acta 1998;1382:191-206.

[22] Hillger F, Herr G, Rudolph R, Schwarz E. Biophysical comparison of BMP-2, ProBMP-2, and the free pro-peptide reveals stabilization of the pro-peptide by the mature growth factor. J Biol Chem 2005;280:14974-80.

[23] Rattenholl A, Lilie H, Grossmann A, Stern A, Schwarz E, Rudolph $\mathrm{R}$. The pro-sequence facilitates folding of human nerve growth factor from Escherichia coli inclusion bodies. Eur J Biochem 2001;268:3296-303.

[24] Arnold K, Bordoli L, Kopp J, Schwede T. The SWISS-MODEL workspace: a Web-based environment for protein structure homology modelling. Bioinformatics 2006;22:195-201.

[25] Guex N, Peitsch MC. SWISS-MODEL and the SwissPdbViewer: an environment for comparative protein modeling. Electrophoresis 1997;18:2714-23.

[26] Schwede T, Kopp J, Guex N, Peitsch MC. SWISS-MODEL: an automated protein homology-modeling server. Nucleic Acids Res 2003;31:3381-5.

[27] Schrodinger LLC. The PyMOL Molecular Graphics System, Version $1.3 \mathrm{r} 1 ; 2010$.

[28] Morgan BA, Fekete DM. Manipulating gene expression with replication-competent retroviruses. Methods Cell Biol 1996; 51:185-218.

[29] Jonk LJC, Itoh S, Heldin CH, ten Dijke P, Kruijer W. Identification and functional characterization of a Smad binding element (SBE) in the JunB promoter that acts as a transforming growth factor-beta, activin, and bone morphogenetic proteininducible enhancer. J Biol Chem 1998;273:21145-52.

[30] Hampf M, Gossen M. A protocol for combined Photinus and Renilla luciferase quantification compatible with protein assays. Anal Biochem 2006;356:94-9.

\section{Glossary}

Brachydactyly: a group of genetically inherited skeletal malformations characterized by the shortening of skeletal elements in the extremities. Brachydactylies are sub-divided into the types A-E.

Chondrodysplasia: a form of dwarfism due to abnormal cartilage development leading to the shortening of the long bones. 
Hamburger Hamilton stage: detailed description of the developmental stages of chicken embryos.

Luciferase assay: a reporter gene assay in which the luciferase expression is controlled by a promoter, for example, a specific binding element that reports the activation of a distinct signaling pathway.
Preproprotein: immature form of a protein that consists of a signal peptide, a proregion and a mature domain.

Symphalangism: a group of genetically inherited skeletal malformations characterized by the fusion of skeletal elements in the extremities. 